\title{
Simultaneous realization of a coherent perfect absorber and laser by zero-index media with both gain and loss
}

\author{
Ping Bai, ${ }^{1,2}$ Kun Ding, ${ }^{3}$ Gang Wang, ${ }^{1}$ Jie Luo, ${ }^{1}$ Zhao-Qing Zhang, ${ }^{3}$ C. T. Chan, ${ }^{3}$ Ying Wu, ${ }^{2,}{ }^{*}$ and Yun Lai ${ }^{1, \dagger}$ \\ ${ }^{1}$ College of Physics, Optoelectronics and Energy and Collaborative Innovation Center of Suzhou Nano Science and Technology, \\ Soochow University, Suzhou 215006, China \\ ${ }^{2}$ Division of Computer, Electrical and Mathematical Sciences and Engineering, King Abdullah University of Science and Technology \\ (KAUST), Thuwal 23955-6900, Saudi Arabia \\ ${ }^{3}$ Department of Physics and Institute for Advanced Study, The Hong Kong University of Science and Technology, Clear Water Bay, \\ Kowloon, Hong Kong, China
}

(Received 14 September 2016; published 19 December 2016)

\begin{abstract}
We investigate a unique type of zero-index medium with both gain and loss (ZIMGL), whose effective permittivity and permeability are both purely imaginary but of opposite signs. We analytically show that, by using a slab of ZIMGL with equal magnitude of loss and gain, simulation realization of a coherent perfect absorber (CPA) and laser, i.e., the so-called CPA laser, can be achieved. Previously the CPA laser was proposed in parity-time $(P T)$ symmetric systems. However, the ZIMGL does not possess the $P T$ symmetry and thus the underlying physics is distinctly different. By designing a photonic crystal (PC) composed of core-shell rods, with loss and gain distributed in either the core or the shell, we have realized such a ZIMGL. The CPA-laser functionality of such a PC is also confirmed in our numerical simulations. Our work provides a different approach for simultaneous realization of CPA and laser besides $P T$-symmetric systems.
\end{abstract}

DOI: 10.1103/PhysRevA.94.063841

\section{INTRODUCTION}

Very recently, parity-time $(P T)$ symmetric systems have attracted intensive research interest. In quantum mechanics, this new system is described by a non-Hermitian Hamiltonian with a complex potential of $V(x)=V^{*}(-x)$, which shares the same eigenstates with the $P T$ operator, and thus can exhibit real eigenvalue spectra. In optics, the $P T$-symmetric system is characterized by complex permittivity exhibiting a symmetric spatial profile of $\varepsilon(x)=\varepsilon^{*}(-x)$ [1-4], i.e., with gain and loss symmetrically distributed in space. Interestingly, the gain in such optical $P T$-symmetric systems plays a far more complicated role than simple loss compensation. Numerous intriguing optical phenomena have been observed in optical $P T$-symmetric systems, such as $P T$-symmetry phase transition and exceptional points [5-8], unidirectional invisibility $[9,10]$, etc. Especially, an optical $P T$-symmetric medium can simultaneously behave as a coherent perfect absorber with $100 \%$ absorption [11-14], and as a laser oscillator $[15,16]$ by simply adjusting the amplitudes and phases of incoming waves. Such a unique device is denoted as a CPA laser [17,18].

In this paper, instead of studying spatially distributed loss and gain, as in $P T$-symmetric systems, we investigate the case where loss and gain are both uniformly distributed in space, but appear in different parameters of a homogeneous medium [19-21]. More specifically, we consider a unique zero-index medium characterized by purely imaginary relative permittivity and relative permeability, i.e., $\varepsilon= \pm|\varepsilon| i$ and $\mu=\mp|\mu| i$, delineating the loss (for positive sign) and gain (for negative sign), respectively. It is interesting to note the fact that the refractive index $n=\sqrt{|\varepsilon||\mu|}$ is a real number, which implies the existence of plane-wave solutions for both

\footnotetext{
*Ying.Wu@kaust.edu.sa

†laiyun@suda.edu.cn
}

electric and magnetic fields. However, as will be demonstrated later, such plane waves do not transport energy because the electric field and magnetic field always differ by a phase of $\pm \pi / 2$, leading to a zero time-averaged Poynting vector. We show explicitly that when a slab of such a medium is embedded in air, the CPA and laser can occur due to the uneven spatial distribution of electric and magnetic fields caused by the boundary condition.

Since the real parts of both $\varepsilon$ and $\mu$ are zero [22-26], we call such a unique type of electromagnetic medium as a zero-index medium with both gain and loss (ZIMGL). Interestingly, we find that the CPA laser can be achieved when $|\varepsilon|=|\mu|$. Since the ZIMGL considered here possesses no $P T$ symmetry, thus our work demonstrates that $P T$ symmetry is not the necessary requirement for the realization of a CPA laser.

To demonstrate the feasibility of our proposal, we design a photonic crystal (PC) which behaves effectively as a ZIMGL. The PC is composed of a square lattice of core-shell dielectric rods, with loss and gain distributed in the permittivities of the cores and shells, respectively. Interestingly, the effective medium of such a PC exhibits a positive purely imaginary permittivity and a negative purely imaginary permeability, which are of the same magnitudes. Therefore, such a PC is an effective ZIMGL. Furthermore, we have numerically demonstrated its functionality as a CPA laser. We have observed the switching between the CPA state with $100 \%$ absorption and the lasing state of stimulated emission, by simply tuning the symmetry of incident waves. These results show that realization of a CPA laser based on ZIMGL is a feasible and promising approach.

\section{THEORETICAL ANALYSIS ON CPA AND LASER BY ZIMGL}

We start our theoretical analysis by considering two counterpropagating plane waves of the same frequency, which 

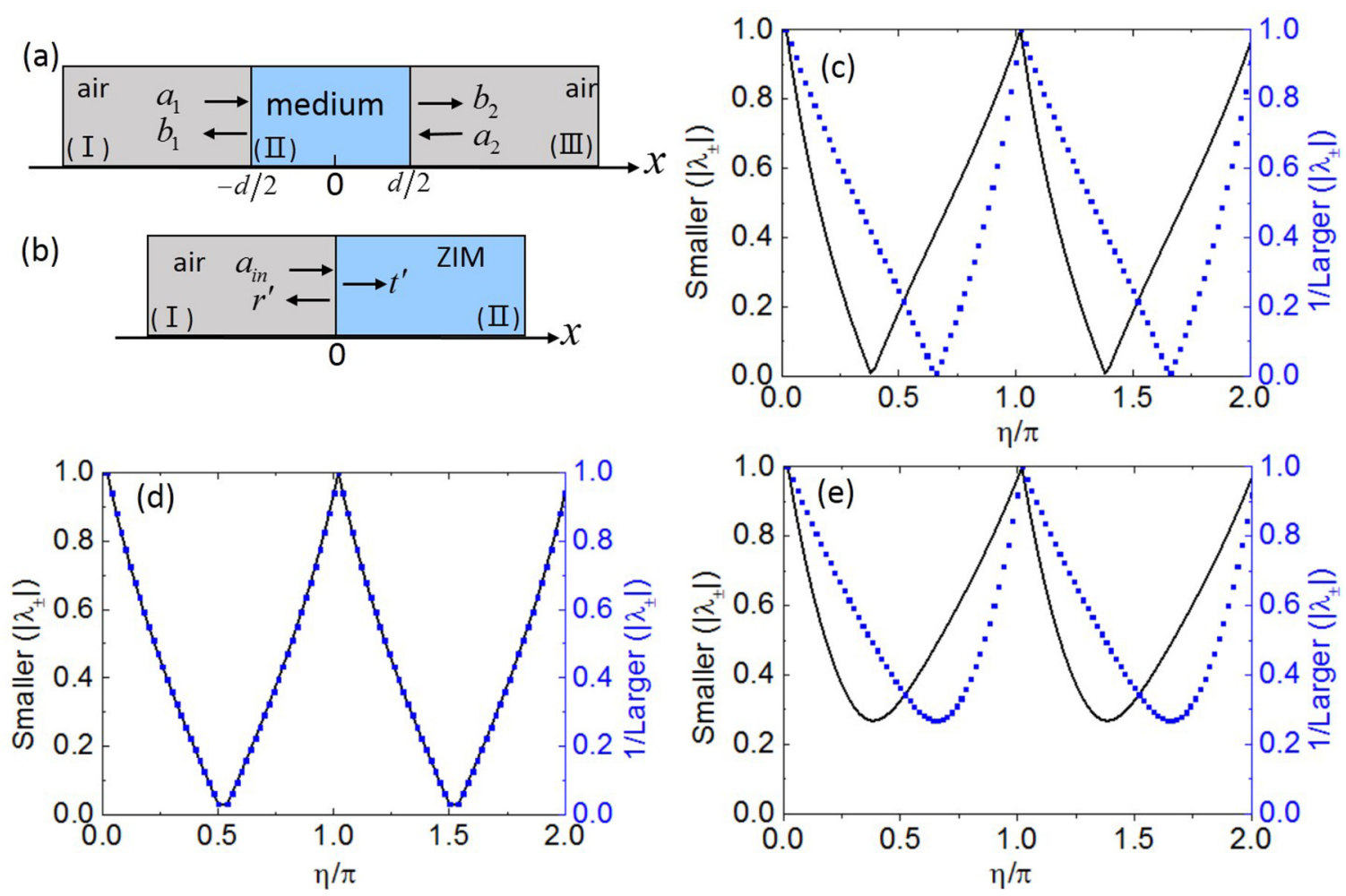

FIG. 1. (a) Schematic graph of the ZIMGL which is homogeneous and symmetric about $x=0$. (b) Schematic graph of the transmission and reflection of plane waves normally incident on a semi-infinite ZIMGL. (c-e) Values of smaller $\left(\left|\lambda_{ \pm}\right|\right)$(black line) and 1/larger $\left(\left|\lambda_{ \pm}\right|\right)($dotted blue line) which are used to determine the poles and zeros of the $\mathbf{S}$ matrix are plotted as functions of $\eta$. The frequency of the incident wave is set to be $f=100 \mathrm{THz}$. The complex permittivity and permeability of the media are set as (c) $\varepsilon=1.2 i, \mu=-0.5 i ;(\mathrm{d}) \varepsilon=1.2 i, \mu=-1.2 i$; (e) $\varepsilon=0.6+1.0392 i, \mu=0.25-0.433 i$, respectively.

are both incident onto a slab of medium. The schematic graph is shown in Fig. 1(a). For simplicity, we have chosen our system to be symmetric about $x=0$. The electric fields in the background (air) and slab can be written as

$$
\begin{aligned}
& E_{1}=a_{1} e^{i k_{0} x}+b_{1} e^{-i k_{0} x} \quad(x \leqslant-d / 2), \\
& E_{2}=a_{2} e^{-i k_{0} x}+b_{2} e^{i k_{0} x} \quad(x \geqslant d / 2), \\
& E_{3}=c e^{i k x}+d e^{-i k x} \quad(-d / 2 \leqslant x \leqslant d / 2),
\end{aligned}
$$

where $k_{0}=\omega / c_{0}, k=k_{0} n . a_{i}, b_{i}, c$, and $d$ are the coefficients of the right and left propagating waves. By matching the standard boundary conditions, it is easy to obtain the following transfer matrix:

$$
\left(\begin{array}{l}
b_{2} \\
a_{2}
\end{array}\right)=\mathbf{M}\left(\begin{array}{l}
a_{1} \\
b_{1}
\end{array}\right)
$$

with $\mathbf{M}=\left(\begin{array}{ll}M_{11} & M_{12} \\ M_{21} & M_{22}\end{array}\right)$ and

$$
\begin{gathered}
M_{11}=e^{-i d k_{0}}\left[\cos (\eta)+i \frac{n^{2}+\mu^{2}}{2 n \mu} \sin (\eta)\right], \\
M_{22}=e^{i d k_{0}}\left[\cos (\eta)-i \frac{n^{2}+\mu^{2}}{2 n \mu} \sin (\eta)\right], \\
M_{12}=i \frac{n^{2}-\mu^{2}}{2 n \mu} \sin (\eta), \\
M_{21}=-M_{12},
\end{gathered}
$$

where $\eta=n k_{0} d$. From Eqs. (3)-(6), it is straightforward to show that $\operatorname{det}(\mathbf{M})=1$.

In terms of scattering matrix $\mathbf{S}$, Eq. (2) can be rewritten as

$$
\left(\begin{array}{l}
b_{2} \\
b_{1}
\end{array}\right)=\mathbf{S}\left(\begin{array}{l}
a_{1} \\
a_{2}
\end{array}\right)=\left(\begin{array}{ll}
t_{1} & r_{2} \\
r_{1} & t_{2}
\end{array}\right)\left(\begin{array}{l}
a_{1} \\
a_{2}
\end{array}\right),
$$

where $t_{1}, t_{2}$ and $r_{1}, r_{2}$ represent the transmission and reflection coefficients of the slab for a plane wave normally incident from the left and right, respectively. By using the condition $\operatorname{det}(\mathbf{M})=1$, we find

$$
t_{1}=t_{2}=t=1 / M_{22} \text { and } r_{1}=r_{2}=r=M_{12} / M_{22} \text {. }
$$

The equalities of $t_{1}=t_{2}$ and $r_{1}=r_{2}$ are expected due to reciprocity and parity symmetry of our system, respectively. In the presence of gain and loss, the system does not have time-reversal symmetry. Here the $\mathbf{S}$ matrix has the following simple form:

$$
\mathbf{S}=\left(\begin{array}{ll}
t & r \\
r & t
\end{array}\right)=\frac{1}{M_{22}}\left(\begin{array}{cc}
1 & M_{12} \\
M_{12} & 1
\end{array}\right),
$$

with eigenvalues $\lambda_{ \pm}=t \pm r=\left(1 \pm M_{12}\right) / M_{22}$ and eigenstates $\varphi_{ \pm}^{T}=(1, \pm 1)$. The two outgoing beams can be obtained from Eqs. (7) and (9) as

$$
\left(\begin{array}{l}
b_{2} \\
b_{1}
\end{array}\right)=\frac{1}{2}\left[\left(a_{1}+a_{2}\right)(t+r) \varphi_{+}+\left(a_{1}-a_{2}\right)(t-r) \varphi_{-}\right] .
$$

In order to obtain CPA, the right-hand side of Eq. (10) has to vanish. This can be achieved either by setting $a_{1}=a_{2}$ 
(in-phase excitation) and finding the condition for $t+r=0$, or by setting $a_{1}=-a_{2}$ (out-of-phase excitation) and finding the condition for $t-r=0$. For lasers the conditions can be determined from the divergence condition of $t+r$ or $t-r$ in the cases of $a_{1}=a_{2}$ or $a_{1}=-a_{2}$, respectively.

Next, we shall focus on the special case of ZIMGL with purely imaginary permittivity and permeability of opposite signs. Such a ZIMGL can be represented by effective parameters of $\varepsilon=i \alpha$ and $\mu=-i \beta$ with $\alpha>0, \beta>0$ or $\alpha<0, \beta<0$. Since the case of $\alpha<0, \beta<0$ only represents a switch between gain and loss (as well as CPA and lasing modes) from the case of $\alpha>0, \beta>0$, we will only consider the case of $\alpha>0, \beta>0$ in the discussions below.

When $\alpha>0, \beta>0$, the condition of $1+M_{12}=0$ is

$$
\frac{\alpha+\beta}{2 \sqrt{\alpha \beta}} \sin (\eta)=1, \eta=\sqrt{\alpha \beta} k_{0} d .
$$

Since $\alpha+\beta>2 \sqrt{\alpha \beta}$, Eq. (11) has two solutions within the region of $0<\eta<\pi$. When $\alpha>\beta>0$, the solution of Eq. (11) with $0<\eta<\pi / 2$ satisfies $M_{22}=\cos (\eta)+\frac{\alpha-\beta}{2 \sqrt{\alpha \beta}} \sin (\eta) \neq 0$. Therefore, we obtain a CPA mode for the in-phase excitation $\left(a_{1}=a_{2}\right)$; i.e., $t+r=\left(1+M_{12}\right) / M_{22}=0$. The other solution of Eq. (11) appearing in the region of $\pi / 2<\eta<\pi$, however, simultaneously satisfies

$$
M_{22}=\cos (\eta)+\frac{\alpha-\beta}{2 \sqrt{\alpha \beta}} \sin (\eta)=0 .
$$

At this solution, we find

$$
t+r=\lim \frac{1+M_{12}}{M_{22}}=e^{-i k_{0} d} \frac{\alpha^{2}-\beta^{2}}{(\alpha-\beta)^{2}-4 \alpha \beta},
$$

which is nonzero when $\alpha \neq \beta$.

It is interesting to point out that the second solution with $M_{22}=0$ and $1-M_{12} \neq 0$ actually corresponds to the lasing mode with two divergent out-of-phase output beams $\left(b_{1}=\right.$ $\left.-b_{2}\right)$ as long as the input beams are not in phase $\left(a_{1} \neq a_{2}\right)$. This can be seen from Eq. (10) with $t-r=\left(1-M_{12}\right) / M_{22}=\infty$. In short, the condition of $1+M_{12}=0$ gives one CPA mode for two in-phase input beams $\left(a_{1}=a_{2}\right)$ and one lasing mode for other input beams, with two out-of-phase output beams $\left(b_{1}=-b_{2}\right)$. The condition of $1-M_{12}=0$ can also be derived similarly, which leads to another set of CPA solutions for the out-of-phase excitation $\left(a_{1}=-a_{2}\right)$, and lasing solutions with two divergent in-phase output beams $\left(b_{1}=b_{2}\right)$ as long as the two input beams are not out of phase $\left(a_{1} \neq-a_{2}\right)$. These CPA and laser modes appear at a different thickness $d$ with a difference $\Delta(d)=\frac{\pi}{k_{0} \sqrt{\alpha \beta}}$ away from the thickness $d$ for the corresponding modes derived earlier from the condition of $1+M_{12}=0$. Thus, by varying the thickness $d$, both CPA and lasing modes repeat themselves with a period of $\Delta(d)=\frac{\pi}{k_{0} \sqrt{\alpha \beta}}$. However, the phases of the two input beams for CPA mode as well as the phases of the two output beams for lasing mode will switch between in phase and out of phase alternatively.

When $\beta>\alpha>0$, the CPA mode occurs in the region of $\pi / 2<\eta<\pi$ and the lasing mode occurs in the region of $0<\eta<\pi / 2$ instead. Their solutions can be obtained from those of the case of $\alpha>\beta>0$ by switching between CPA and laser modes.
For the cases of $\alpha \neq \beta$ discussed so far, CPA and lasing modes cannot occur simultaneously in a single slab. A numerical demonstration is shown in Fig. 1(c) for the case of $\varepsilon=1.2 i$ and $\mu=-0.5 i$. In Fig. 1(c), we fix the frequency at $f=100 \mathrm{THz}$ and calculate the two eigenvalues of the $\mathbf{S}$ matrix, $\lambda_{ \pm}$, as a function of $\eta$. The absolute value of the smaller eigenvalue is plotted by a black line and the inverse of the larger eigenvalue by a dotted blue line. The CPA (lasing) mode is achieved when the black (dotted blue) line touches the $\eta$ axis. Two CPA modes with $\eta=0.367 \pi, 1.367 \pi$ and two lasing modes with sample thicknesses $\eta=0.632 \pi, 1.632 \pi$ are shown in Fig. 1(c). The first and second CPA modes correspond to the in-phase and out-of-phase input beams, respectively.

However, when $\alpha=\beta$, interesting things happen. From Eqs. (11) and (12), the conditions of CPA and lasing modes obtained from $1+M_{12}=0$ both turn into $\eta=\pi / 2[\sin (\eta)=1$ and $\cos (\eta)=0$ ] within the region of $0<\eta<\pi$. Interestingly, from Eq. (13), it can be seen that when $\alpha=\beta$, we have $t+r=0$ even though $M_{22}=0$, indicating the realization of CPA. Therefore, CPA and lasing modes can be simultaneously realized, i.e., the so-called CPA laser.

The coexistence of CPA and laser can also be easily seen from the determinant of the $\mathbf{S}$ matrix, i.e.,

$$
|\operatorname{det}(\mathbf{S})|=\left|\lambda_{+} \lambda_{-}\right|=\left|\frac{2 i n \mu \cos \eta-\left(n^{2}+\mu^{2}\right) \sin \eta}{2 i n \mu \cos \eta+\left(n^{2}+\mu^{2}\right) \sin \eta}\right| .
$$

When $\alpha=\beta$, we have $n^{2}+\mu^{2}=0$ and $|\operatorname{det}(\mathbf{S})|=1$. The CPA laser occurs when $\cos (\eta)=0$ at which both numerator and denominator of $\mathbf{S}$ become zero, giving rise to the CPA and laser simultaneously. When $\alpha \neq \beta,|\operatorname{det}(\mathbf{S})|$ has different expressions for the numerator and denominator and the zeros (CPA) and poles (lasing) occur at different sample thicknesses. As a demonstration of this case, in Fig. 1(d), we plot only the absolute value of the smaller eigenvalue of the $\mathbf{S}$ matrix as a function of sample thickness for the case of $\varepsilon=1.2 i, \mu=$ $-1.2 i$. The inverse of the larger eigenvalue follows the same curve because $\left|\lambda_{+} \lambda_{-}\right|=1$. The overlap of poles and zeros in this case implies that the CPA and laser can be simultaneously achieved in a single slab. However, at each CPA-laser solution, the phase relation between two input CPA beams will switch alternately from in phase to out of phase or vice versa when the next solution is reached. At the same time, the phase relation between two output laser beams will switch from out of phase to in phase or vice versa.

Finally, we consider the general case of complex $\varepsilon$ and $\mu$ with a real refractive index, e.g., $\varepsilon=0.6+1.0392 i$ and $\mu=0.25-0.433 i$. Here the refractive index is a real number $n=0.7746$. The results in Fig. 1(e) show that neither CPA nor laser can occur at any sample thickness. This can also be understood from Eq. (14). For such a medium with a real refractive index, both the numerator and denominator of $\operatorname{det}(\mathbf{S})$ are complex functions of frequency and they do not vanish for any real frequency.

Now, we study the behaviors and properties of various wave functions in ZIMGL. We should first point out that the lasing mechanism in our system is very different from that of ordinary laser systems. To see this, we consider a plane wave normally incident on a semi-infinite ZIMGL from one side, as illustrated in Fig. 1(b). The electric and magnetic fields in region I can 
be written as

$$
\begin{aligned}
& E_{\mathrm{I}}=e^{i k_{0} x}+r^{\prime} e^{-i k_{0} x}, \\
& H_{\mathrm{I}}=e^{i k_{0} x}-r^{\prime} e^{-i k_{0} x} .
\end{aligned}
$$

Here $r^{\prime}$ is the reflection coefficient. Here we have assumed that the polarizations of electric and magnetic fields are along the $y$ and $z$ directions, respectively. The electric and magnetic fields in the ZIMGL are obtained as

$$
\begin{aligned}
& E_{\mathrm{II}}=t^{\prime} e^{i k x}, \\
& H_{\mathrm{II}}=t^{\prime} \frac{k}{k_{0} \mu} e^{i k x},
\end{aligned}
$$

where $t^{\prime}$ is the transmission coefficient. By matching the boundary conditions, we obtain

$$
\begin{aligned}
& 1+r^{\prime}=t^{\prime}, \\
& 1-r^{\prime}=-i t^{\prime} \frac{1}{\xi},
\end{aligned}
$$

where $\xi=\sqrt{\frac{|\mu|}{|\varepsilon|}}$. We further find that the reflection coefficient can be simplified as

$$
r^{\prime}=\frac{-1+i \xi}{1+i \xi}
$$

An interesting conclusion drawn from Eq. (18) is that $\left|r^{\prime}\right|=$ 1 , indicating total reflection of the waves. Therefore, there is no flux going into the ZIMGL. This is different from ordinary laser systems, which always have some nonzero transmission; i.e., $\left|t^{\prime}\right| \neq 0$. The absence of energy flux in ZIMGL can also be seen from the vanishing time-averaged Poynting vector; i.e.,

$$
\langle S(x)\rangle_{t}=\frac{1}{2} \operatorname{Re}\left(E_{\mathrm{II}} H_{\mathrm{II}}^{*} \hat{y} \times \hat{z}\right)_{x}=\operatorname{Re}\left(\frac{i}{2 \xi}\left|t^{\prime}\right|^{2}\right)=0,
$$

due to $\pi / 2$ phase difference between the electric and magnetic fields as shown in Eq. (16).

Now we show the field patterns of CPA modes. For the case of two in-phase input beams, the condition of CPA modes is $n k_{0} d=\frac{\pi}{2}+2 M \pi$. The normalized electric and magnetic fields in each region have the following forms:

$$
\begin{aligned}
E_{\mathrm{I}} & =e^{i k_{0} x}, \quad H_{\mathrm{I}}=e^{i k_{0} x}, \\
E_{\mathrm{II}} & =\sqrt{2} \cos \left(n k_{0} x\right), \quad H_{\mathrm{II}}=-\sqrt{2} \sin \left(n k_{0} x\right), \\
E_{\mathrm{III}} & =e^{-i k_{0} x}, \quad H_{\mathrm{III}}=-e^{-i k_{0} x} .
\end{aligned}
$$

The corresponding time-averaged Poynting vector has the following pattern:

$$
\langle S(x)\rangle_{t}= \begin{cases}\frac{1}{2} & x \in \mathrm{I} \\ -\frac{1}{2} \sin \left(2 n k_{0} x\right) & x \in \mathrm{II} . \\ -\frac{1}{2} & x \in \mathrm{III}\end{cases}
$$

A typical curve of Eq. (21) is shown in Fig. 2(a) for $M=0$. It is clearly shown that the energy that flows in ZIMGL from the background is totally absorbed.

For the lasing mode, the electric and magnetic fields in each region are

$$
\begin{aligned}
E_{\mathrm{I}} & =r_{t} e^{-i k_{0} x}, \quad H_{\mathrm{I}}=-r_{t} e^{-i k_{0} x}, \\
E_{\mathrm{II}} & =-\sqrt{2} r_{t} \sin \left(n k_{0} x\right), \quad H_{\mathrm{II}}=-\sqrt{2} r_{t} \cos \left(n k_{0} x\right), \\
E_{\mathrm{III}} & =-r_{t} e^{i k_{0} x}, \quad H_{\mathrm{III}}=-r_{t} e^{i k_{0} x},
\end{aligned}
$$
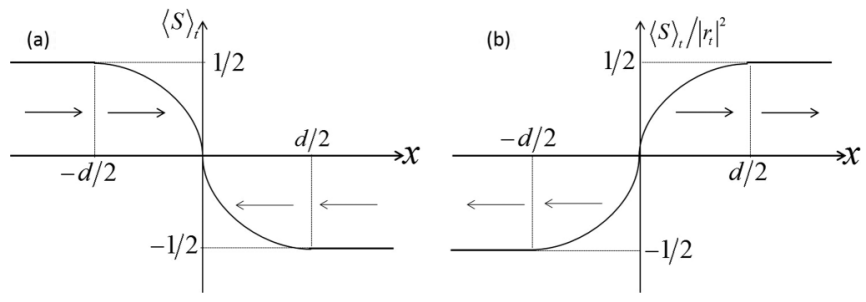

FIG. 2. The time-averaged Poynting vector distribution for ZIMGL in one-dimensional structure [see Fig. 1(a)] (a) in CPA mode and (b) in lasing mode.

where $r_{t}$ denotes the amplitude of the output beams. Since $r_{t}$ diverges at the lasing mode, we have ignored the source term in Eq. (22). The corresponding time-averaged Poynting vector now becomes

$$
\langle S(x)\rangle_{t}= \begin{cases}-\frac{1}{2}\left|r_{t}\right|^{2} & x \in \mathrm{I} \\ \frac{1}{2} \sin \left(2 n k_{0} x\right)\left|r_{t}\right|^{2} & x \in \mathrm{II} . \\ \frac{1}{2}\left|r_{t}\right|^{2} & x \in \mathrm{III}\end{cases}
$$

A typical curve for the normalized Poynting vector $\langle S(x)\rangle_{t} /\left|r_{t}\right|^{2}$ for $M=0$ is shown in Fig. 2(b). It is interesting to show that Eqs. (20)-(23) satisfy the following steady-state Poynting theorem for harmonic fields:

$$
\frac{d}{d x}\langle S(x)\rangle_{t}=-\frac{k_{0}}{2}\left[\operatorname{Im} \varepsilon|E|^{2}+\operatorname{Im} \mu|H|^{2}\right] .
$$

In the case of CPA, by taking $\operatorname{Im} \varepsilon=-\operatorname{Im} \mu=\alpha=n$ and using Eqs. (20) and (21) in Eq. (24), it is easy to show that both sides of Eq. (24) give $\alpha k_{0} \cos \left(2 n k_{0}\right)$. A similar case is obtained for the laser. Equations (22) and (23) give $\alpha k_{0} \cos \left(2 n k_{0}\right)\left|r_{t}\right|^{2}$ on both sides of Eq. (24). The generation of the Poynting vector in ZIMGL is due to the uneven spatial distributions of the electric and magnetic fields inside ZIMGL, which in turn acts as a net source or sink of the Poynting vector at every point. At the CPA laser, the behaviors of $\langle S(x)\rangle_{t}$ are identical for CPA and laser except opposite in sign.

Now we show the field patterns for the other set of CPAlaser modes at $n k_{0} d=\frac{3 \pi}{2}+2 M \pi$. The electric and magnetic fields now become

$$
\begin{aligned}
E_{\mathrm{I}} & =e^{i k_{0} x}, \quad H_{\mathrm{I}}=e^{i k_{0} x}, \\
E_{\mathrm{II}} & =\sqrt{2} \sin \left(n k_{0} x\right), \quad H_{\mathrm{II}}=-\sqrt{2} \cos \left(n k_{0} x\right), \\
E_{\mathrm{III}} & =-e^{-i k_{0} x}, \quad H_{\mathrm{III}}=e^{-i k_{0} x},
\end{aligned}
$$

and

$$
\begin{aligned}
E_{\mathrm{I}} & =r_{t} e^{-i k_{0} x}, \quad H_{\mathrm{I}}=-r_{t} e^{-i k_{0} x}, \\
E_{\mathrm{II}} & =\sqrt{2} r_{t} \cos \left(n k_{0} x\right), \quad H_{\mathrm{II}}=\sqrt{2} r_{t} \sin \left(n k_{0} x\right), \\
E_{\mathrm{III}} & =r_{t} e^{i k_{0} x}, \quad H_{\mathrm{III}}=r_{t} e^{i k_{0} x},
\end{aligned}
$$

respectively. Although the field patterns of the two CPA-laser modes are different, the Poynting vector distributions are unchanged and are described by Fig. 2.

\section{DESIGN OF ZIMGL}

The theoretical analysis in Sec. II shows that a slab of ZIMGL with the same magnitude for $\varepsilon$ and $\mu$ can realize 

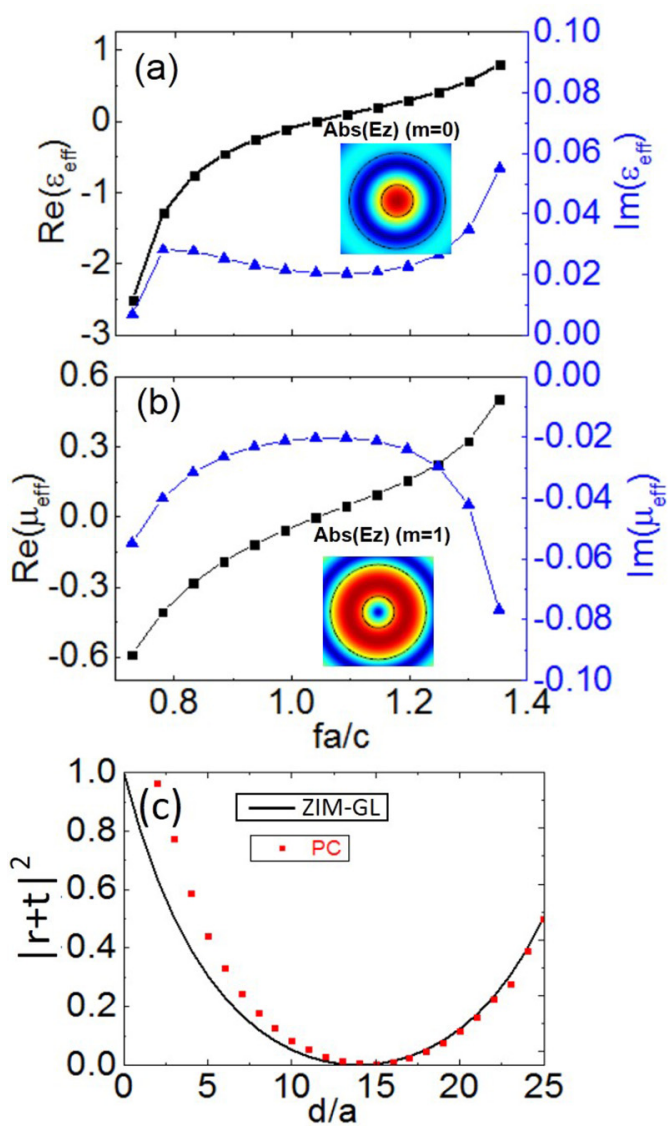

FIG. 3. (a,b) The real (black square line) and imaginary (blue triangle line) parts of effective permittivity $\varepsilon_{\text {eff }}$ and permeability $\mu_{\text {eff }}$ of the designed PC structure as functions of frequency, respectively. The core-shell structure with inner and outer radii of $r_{1}=0.16 a, r_{2}=$ $0.48 a$ is designed, where $a$ is the lattice constant of the square lattice. The permittivities of the core and shell are set to be $\varepsilon_{1}=1.133+$ $0.11 i, \varepsilon_{2}=1.07-0.054 i$, respectively. The dashed lines indicate the CPA-laser point of $f a / c=1.04, \varepsilon_{\text {eff }}=0.02 i$, and $\mu_{\text {eff }}=-0.02 i$. The insets in $(a, b)$ show the field distributions of the electric fields (absolute value) under the excitation of monopolar and dipolar waves, respectively. (c) The analytical outgoing spectra $|r+t|^{2}$ as a function of the varying thicknesses $d$ of the ZIMGL (black line), and the designed PC structure (red square), under in-phase incidence from both sides.

both CPA and laser simultaneously. However, no naturally occurring material can satisfy such a stringent requirement on the material parameters. In this section, we propose a design method, guided by an effective medium approximation that has been generally applied to metamaterials and PCs [27-32], to realize a ZIMGL by using a PC composed of an array of core-shell structures. A schematic is shown in the inset of Fig. 3; a lossy core material with radius $r_{1}$ is coated by a layer of gain material with radius $r_{2}$ and arranged in a square lattice structure. The permittivities of the core and shell are denoted by the complex dielectric constants $\varepsilon_{1}$ and $\varepsilon_{2}$, respectively. In order to obtain the appropriate parameters of $\varepsilon_{1}, \varepsilon_{2}$ as well as the corresponding radii $r_{1}, r_{2}$ for the effective ZIMGL, we apply a systematic inverse design method based on an effective medium theory [33]. The details can be found in Appendix A.

We set the working frequency as $f=100 \mathrm{THz}$, at which the medium exhibits effective parameters of $\varepsilon_{\text {eff }}=0.02 i$ and $\mu_{\text {eff }}=-0.02 i$. According to the previous analysis, such a medium with the thickness $d=43.673 \mu \mathrm{m}$ can act as a CPA laser, i.e., the simultaneous realization of CPA and laser. The lattice constant is obtained by assuming there are 14 periods along the propagation direction, i.e., $a=d / 14=3.119 \mu \mathrm{m}$, and the inner and outer radii of the core-shell cylinder are set to be $r_{1}=0.16 a, r_{2}=0.48 a$, respectively. Based on the effective medium theory, we can obtain the complex permittivities for the core and shell of cylinders: $\varepsilon_{1}=1.33+0.11 i$ and $\varepsilon_{2}=1.07-0.054 i$ (see Appendix A for details). In Fig. 3 we plot the real (black square line) and imaginary (blue triangle line) parts of permittivity and permeability in a frequency range according to the following effective medium formulas (see Ref. [33]):

$$
\begin{gathered}
\frac{\varepsilon_{\text {eff }}+2 \varepsilon_{0} \frac{J^{\prime}{ }_{0}\left(k_{0} r_{0}\right)}{k_{0} r_{0} J_{0}\left(k_{0} r_{0}\right)}}{\varepsilon_{\text {eff }}+2 \varepsilon_{0} \frac{Y_{0}^{\prime}{ }_{0}\left(k_{0} r_{0}\right)}{k_{0} r_{0} Y_{0}\left(k_{0} r_{0}\right)}}=\frac{Y_{0}\left(k_{0} r_{0}\right)}{i J_{0}\left(k_{0} r_{0}\right)}\left(\frac{D_{0}}{1+D_{0}}\right), \\
\frac{\mu_{\text {eff }}-\mu_{0} \frac{J_{1}\left(k_{0} r_{0}\right)}{k_{0} r_{0} J^{\prime}{ }_{1}\left(k_{0} r_{0}\right)}}{\mu_{\text {eff }}-\mu_{0} \frac{Y_{1}\left(k_{0} r_{0}\right)}{k_{0} r_{0} Y^{\prime}{ }_{1}\left(k_{0} r_{0}\right)}}=\frac{Y^{\prime}{ }_{1}\left(k_{0} r_{0}\right)}{i J^{\prime}{ }_{1}\left(k_{0} r_{0}\right)}\left(\frac{D_{1}}{1+D_{1}}\right),
\end{gathered}
$$

where $J_{m}(x)$ and $Y_{m}(x)(m=0,1)$ are the Bessel function of the first and second kind, respectively. $D_{m}$ represent the Mie scattering coefficients of the core-shell cylinder. As shown in Figs. 3(a) and 3(b), the real parts of $\varepsilon_{\text {eff }}$ and $\mu_{\text {eff }}$ vanish simultaneously at frequency $f a / c=1.04$, where their imaginary parts are close to $\operatorname{Im}\left(\varepsilon_{\text {eff }}\right)=0.02 i$ and $\operatorname{Im}\left(\mu_{\text {eff }}\right)=$ $-0.02 i$. Such a combination of effective medium parameters satisfies the condition of CPA laser. The field distributions (absolute value of $E_{Z}$ ) of the core-shell cylinder under the excitation of monopolar and dipolar waves are displayed in the insets of Figs. 3(a) and 3(b), where dark red represents the maximum value and dark blue represents zero. They correspond to the eigenstates of the structure. It is clearly seen that under the monopolar excitation $(m=0)$, the fields are concentrated in the lossy core, and therefore the majority of the incident wave energy is absorbed by the core; while under the dipolar excitation, the fields are concentrated in the shell with gain medium, thus implying energy enhancement by the gain medium in the shell. In order to further improve the accuracy of the effective parameters beyond the limitation of the effective medium theory, we have performed band structure calculations based on a finite element software, COMSOL MULTIPHYSICS, and plotted the results in Appendix B. By fine-tuning the parameters of the core and shell, we finally obtain an effective medium which almost exactly satisfies the requirement of CPA laser at a real frequency. The final values of the parameters are $\varepsilon_{1}=1.154+0.11 i$ and $\varepsilon_{2}=1.07-0.054 i$, which give rise to effective permittivity and permeability of $\varepsilon_{\text {eff }}=0.01838 i$ and $\mu_{\text {eff }}=-0.01838 i$ at the real frequency of $f a / c=0.972$. In the next section, we will conduct numerical simulations to verify the functionality of the designed PC as the required effective ZIMGL. 

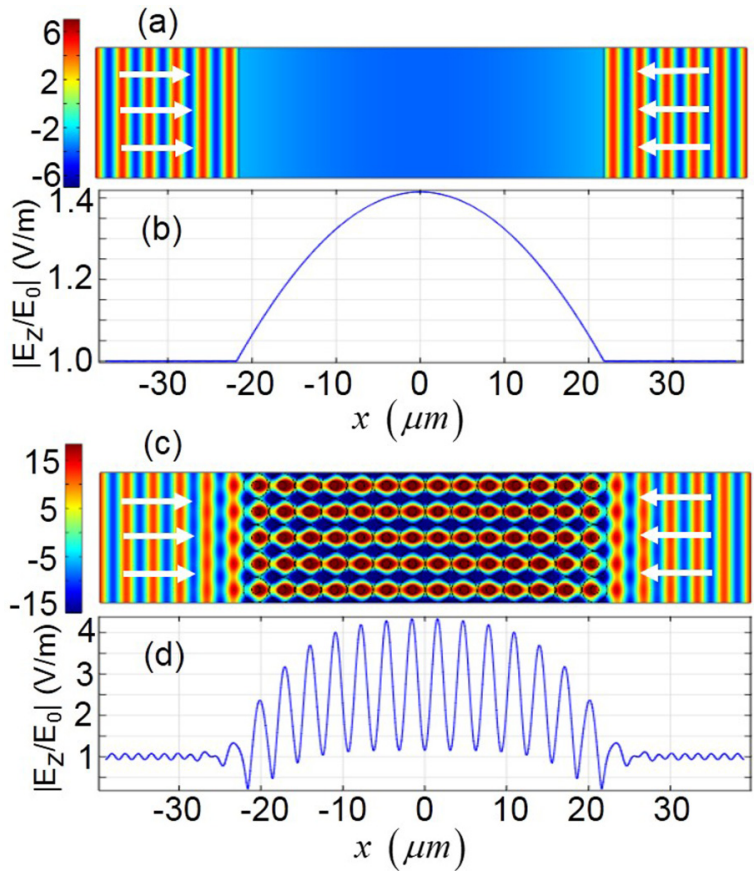

FIG. 4. (a,c) show the field distributions of electric field $E_{z}$ in a slab of the effective medium and the real structure of the ZIMGL in the case of $\Delta \varphi=0$, respectively. The arrows represent the power flow in the background. $(b, d)$ show the normalized amplitude of electric field $\left|E_{z} / E_{0}\right|$ for the cases of (a,c), respectively.

\section{NUMERICAL SIMULATIONS OF CPA LASER}

The scattering property of a slab of the core-shell cylinder structures proposed in Sec. III is simulated by COMSOL. Figure 3(c) shows the outgoing spectra $|r+t|^{2}$ with varying thicknesses $d$ of the effective medium of ZIMGL (Black line) and the real structure (Red square) under the excitation of two counter-propagating coherent waves with the same phase. It is seen that the two sets of results agree with each other, which verifies the functionality of the designed $\mathrm{PC}$ as an effective ZIMGL in terms of transmission properties. From Fig. 3(c), we found that when the thickness of the slab is around $14 a$, the outgoing waves vanish, indicating CPA.

The effects of the CPA and laser by using the real PC structure is demonstrated in Figs. 4(c) and 5(c), which show the field distributions of electromagnetic wave $E_{z}$, when two coherent counter-propagating incoming waves are normally incident on the structure with phase difference $\Delta \varphi=0$ (CPA) and $\Delta \varphi=\pi$ (laser), respectively. The corresponding results obtained from the effective medium of ZIMGL are shown in Figs. 4(a) and 5(a). In the case of $\Delta \varphi=0$, nearly all the incident waves are absorbed by such a slab of ZIMGL, as indicated by the total power flow (denoted by the arrows) in Figs. 4(a) and 4(c). In Figs. 4(b) and 4(d), we also plot the amplitudes (normalized to the incident wave) of the electric field for the cases corresponding to Figs. 4(a) and 4(c), respectively. It is clearly seen that for both cases, the electric field in the background is nearly unity, meaning there is no reflection and that almost all of the incident energy is absorbed.
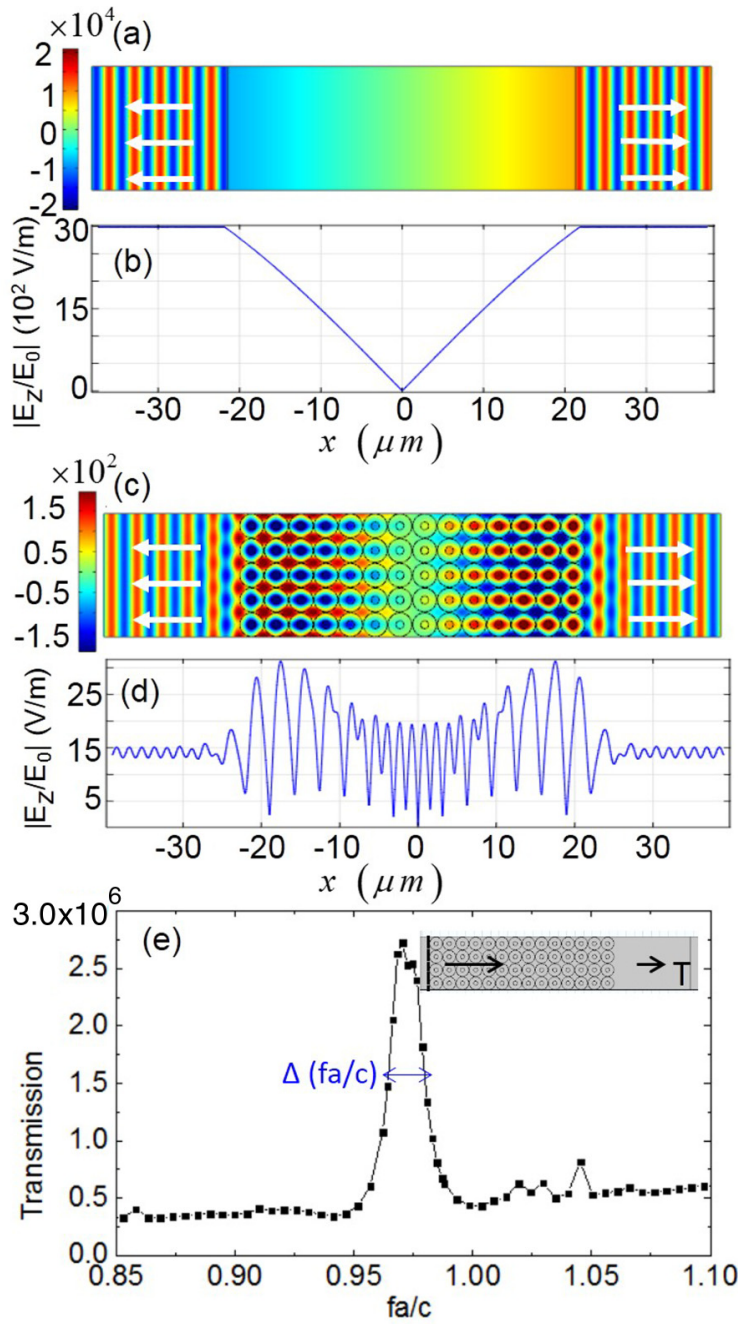

FIG. 5. (a,c) show, respectively, the field distributions of electric field $E_{z}$ in a slab of the effective medium and the real structures of the ZIMGL in the case of $\Delta \varphi=\pi$. The arrows represent the power flow in the background. (b,d) show the normalized amplitude of electric field $\left|E_{z} / E_{0}\right|$ for the cases of (a,c), respectively. (e) Transmission as a function of the frequency for a model with the schematic graph shown in the inset in (e).

In the case of $\Delta \varphi=\pi$, however, the power flows are completely reversed, as illustrated in Figs. 5(a) and 5(c). The normalized amplitudes of the electric field in the background shown in Figs. 5(b) and 5(d) are found to be about $3 \times 10^{3}$ (in effective medium of ZIMGL) and 15 (in real structure of ZIMGL) times of the incident waves, indicating a lasing phenomenon. On the other hand, in order to further confirm the lasing mode in photonic structure, we put a row of point sources with line current at the edge of the PC. The structure is displayed in the insets of Fig. 5(e). The transmission is significantly amplified in the lasing mode and verifies energy enhancement. Figure 5(e) show the transmission as a function of frequency. A narrow peak is observed in the frequency spectrum with $\Delta(f a / c)=0.021$, at the frequency $\mathrm{fa} / \mathrm{c}=0.972$ for the ideal lasing mode. Through Figs. 4 and 5, the functionality of CPA-laser is verified. 


\section{DISCUSSION AND CONCLUSION}

In above paragraphs, we have proposed a PC composed of core-shell structures with gain in shell and loss in core to realize the unique material of ZIMGL. Since the magnitude of loss and gain are both small, they can be realized by using conventional materials that has been generally applied to fabricate the $P T$-symmetric structures. For instance, the lossy core may be fabricated by metals with loss in permittivity. The shell with weak gain can be achieved by using the conventional complementary meta-oxide-semiconductor silicon technology [9]. Or, the gain or loss in PC may be introduced by introducing $\mathrm{Cr}$ or Ge material [34] into the shell or core. We note that the amount of loss or gain should be controlled precisely so as to realize ZIMGL with balanced loss and gain, just like $P T$-symmetric systems.

In conclusion, we introduce a unique concept of ZIM with its permittivity and permeability being purely imaginary, but exhibiting opposite signs. We demonstrate, through rigorous derivations, that such a ZIM can achieve CPA and laser effect. Especially, when the loss and gain have the same magnitude, the so-called CPA laser, i.e., simultaneous realization of CPA and laser which was previously only observed in $P T$-symmetric systems or non- $P T$-symmetric bilayer materials [35], can be realized in our system, which exhibits no PT symmetry. The functionalities of CPA and laser have been studied in Ref. [36] and they can be chosen by the way of excitation, e.g., the symmetry of incident waves hereby. Interestingly, our system exhibits the cross-matched $P T$ symmetry, i.e., $\varepsilon(x)=\mu^{*}(-x)$ [37].

We also propose a practical scheme to design a ZIMGL by using PC structures. By employing the effective medium theory in an inverse manner, and we have successfully designed a square lattice of core-shell dielectric cylinders whose effective medium parameters meet the requirements of the CPA-laser. Numerical simulations verify the fascinating functionality of switching between a CPA and a laser by changing the symmetry of the excitations, which is consistent with analytical results. Our work demonstrates intriguing possibilities in non-Hermitian optic systems with gain and loss distributed in different parameters.

\section{ACKNOWLEDGMENTS}

This work was supported by the State Key Program for Basic Research of China (Grants No. 2014CB360505 and No. 2012CB921501), National Natural Science Foundation of China (Grants No. 11374224 and No. 61671314), King Abdullah University of Science and Technology, a Project Funded by the Priority Academic Program Development of Jiangsu Higher Education Institutions (PAPD), and the Hong Kong Research Grants Council (Grant No. AoE/P-02/12).

\section{APPENDIX A: THE INVERSE DESIGN TO REALIZE THE ZIMGL}

In this part, we give the derivation of the inverse design to realize the ZIMGL based on effective medium theory in two dimensions. The system considered here is a composite consisting of a periodic array of core-shell cylinders possessing complex dielectric permittivities $\varepsilon_{1}, \varepsilon_{2}$ and magnetic

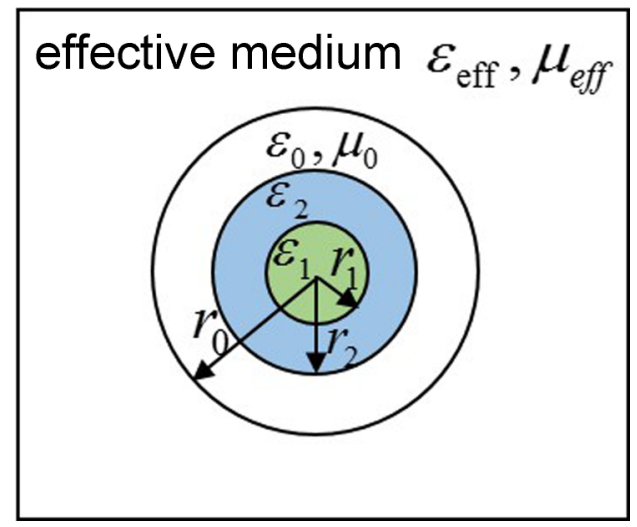

FIG. 6. The microstructure of the composite.

permeabilities $\mu_{1}=\mu_{2}=1$ with radii $r_{1}, r_{2}$, embedded in air. The microstructure of the composite as shown in Fig. 6.We consider only the case of transverse electric (TE) waves, the electric field is parallel to the cylinder and its solutions in each region can be written as

$$
\begin{aligned}
& E_{e}(r)=\sum_{m}\left[a_{e m} J_{m}\left(k_{e} r\right)+b_{e m} H_{m}^{(1)}\left(k_{e} r\right)\right] e^{i m \theta} \quad\left(r \geqslant r_{0}\right), \\
& E_{0}(r)=\sum_{m}\left[a_{0 m} J_{m}\left(k_{0} r\right)+b_{0 m} H_{m}^{(1)}\left(k_{0} r\right)\right] e^{i m \theta} \quad\left(r_{2} \leqslant r \leqslant r_{0}\right), \\
& E_{1}(r)=\sum_{m}\left[a_{1 m} J_{m}\left(k_{1} r\right)+b_{1 m} H_{m}^{(1)}\left(k_{1} r\right)\right] e^{i m \theta} \quad\left(r_{1} \leqslant r \leqslant r_{2}\right), \\
& E_{2}(r)=\sum_{m} a_{2 m} J_{m}\left(k_{2} r\right) e^{i m \theta}\left(r \leqslant r_{1}\right),
\end{aligned}
$$

where $k_{0}=\omega / c_{0}, k_{1}=k_{0} \sqrt{\varepsilon_{1}}, k_{2}=k_{0} \sqrt{\varepsilon_{2}}, k_{e}=k_{0} \sqrt{\varepsilon_{e}} \sqrt{\mu_{e}}$. Here $J_{m}(x)$ and $H_{m}^{(1)}(x)$ are the Bessel function and Hankel function of the first kind, respectively. By matching the standard boundary conditions on the interfaces $r=r_{1}, r_{2}$, we can obtain

$$
\frac{b_{0 m}}{a_{0 m}}=D_{m}\left(\varepsilon_{1}, \varepsilon_{2}\right),
$$

where $D_{m}\left(\varepsilon_{1}, \varepsilon_{2}\right)$ represent the Mie scattering coefficients of the core-shell cylinder. Based on the effective medium theory as $b_{e m}=0$ and standard boundary conditions on the interface $r=r_{0}$, we can get

$$
\frac{b_{0 m}}{a_{0 m}}=S_{m}\left(\varepsilon_{e}, \mu_{e}\right) .
$$

Obviously, from Eqs. (A2) and (A3),

$$
D_{m}\left(\varepsilon_{1}, \varepsilon_{2}\right)=S_{m}\left(\varepsilon_{e}, \varepsilon_{e}\right), \quad(m=0,1,2, \ldots) .
$$

Here we determine the effective parameters we want to achieve are $\varepsilon_{\mathrm{e}}=0.02 i, \mu_{e}=-0.02 i$ and the incident waves with frequency $f=100 \mathrm{THz}$; thus the effective wave number in the medium is a real value as $k_{e}=0.042 \mathrm{rad} / \mathrm{m}$ which likely is without gain or loss. According to conditions of CPA and laser as shown in our paper, this material with sample thickness $d=43.673 \mathrm{~m}$ can act as a CPA laser. Then we might as well make this thickness equal to 14 times the lattice constant of the photonic crystal; i.e., $a=d / 14=3.1195 \mathrm{~m}$. When the 

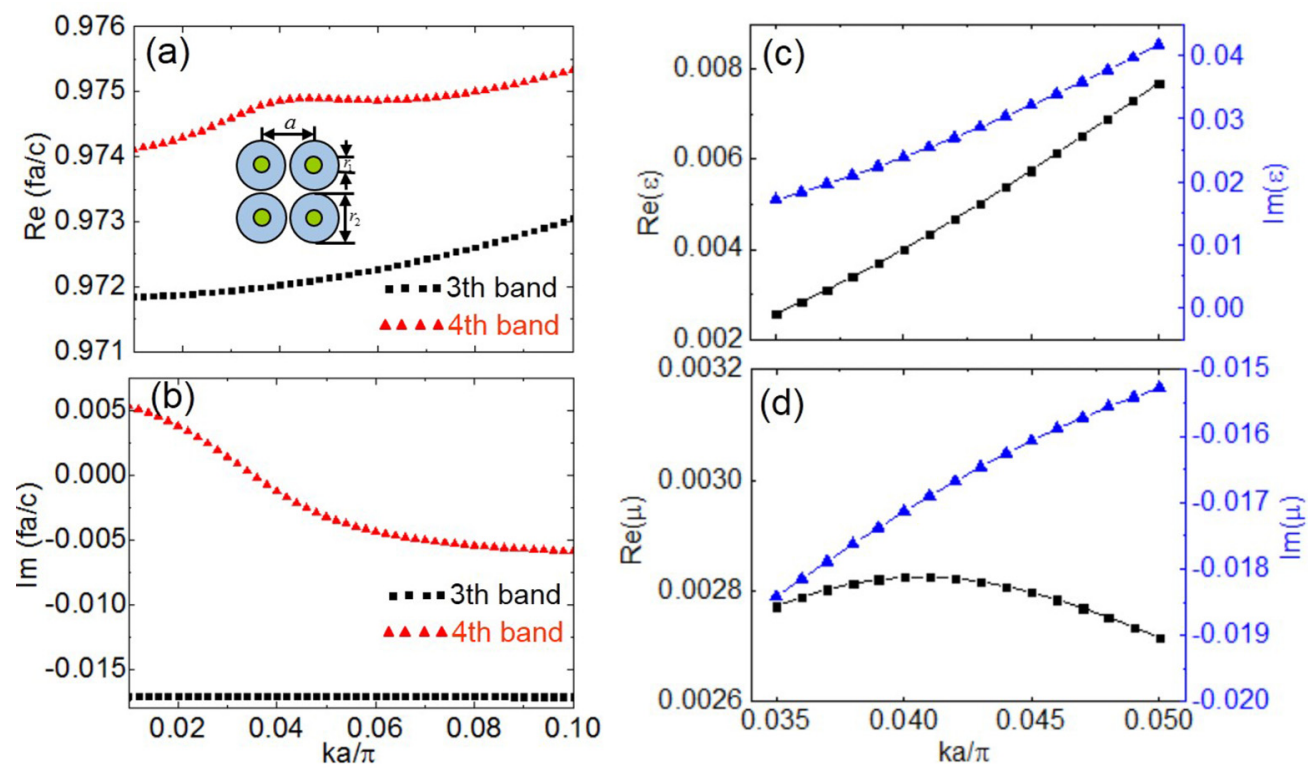

FIG. 7. The band structure of the designed photonic crystal with a square lattice before tuning. (a, b) the real and imaginary part of eigenfrequency in third (black square) and fourth (red triangle) bands near the $\Gamma$ point for a core-shell PC constructed of core cylinders with radius $r_{1}=0.16 a$, relative permittivity $\varepsilon_{1}=1.33+0.11 i$, and shell cylinders with radius $r_{2}=0.48 a$, permittivity $\varepsilon_{2}=1.07-0.054 i$. The permeabilities of core and shell are both $\mu=1$. (c,d) show the real (black square line) and imaginary (blue triangle line) parts of effective permittivities and permeabilities corresponding to the eigenvalues.

radii of the cylinders are given as $r_{1}=0.16 a, r_{2}=0.48 a$, then we achieved the permittivities of the cylinders as $\varepsilon_{1}=$ $1.33+0.11 i, \varepsilon_{2}=1.07-0.054 i$ with both gain and loss from Eq. (A4) with $m=0$ and $m=1$.

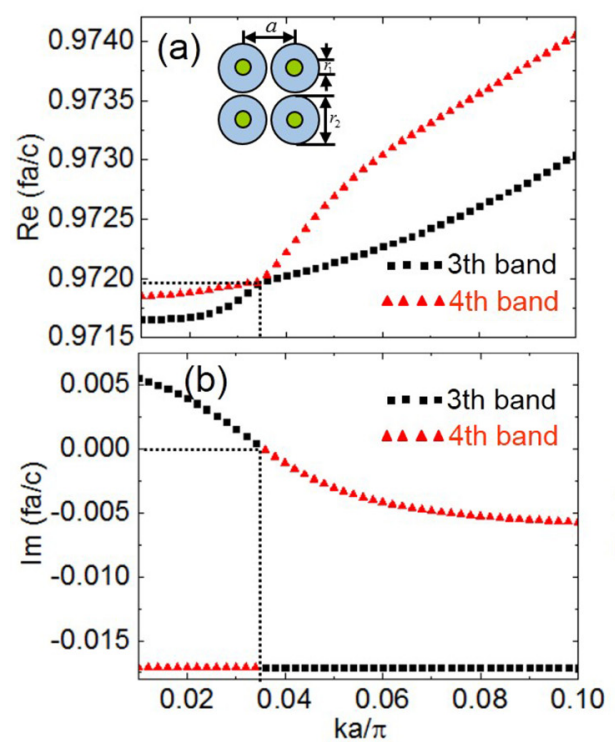

\section{APPENDIX B: THE BAND STRUCTURE OF OUR DESIGNED PHOTONIC CRYSTAL}

In case of TE waves with electric fields polarized in the $z$ direction, the interface of the PC and air is in the $y z$ plane.

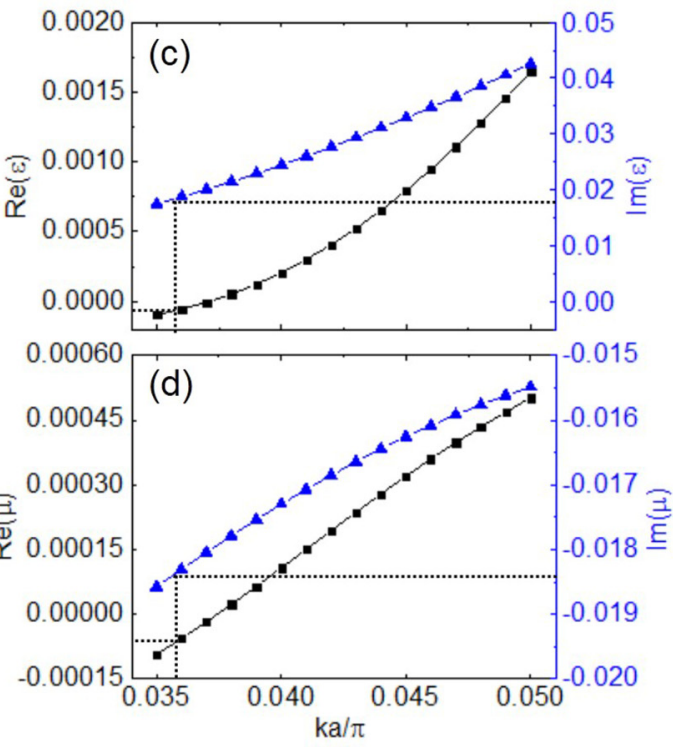

FIG. 8. The band structure of the fine-tuned photonic crystal. (a,b) the real and imaginary part of eigenfrequency in third (black square) and fourth (red triangle) bands near the $\Gamma$ point for a core-shell PC constructed of core cylinders with radius $r_{1}=0.16 a$, relative permittivity $\varepsilon_{1}=1.154+0.11 i$, and shell cylinders with radius $r_{2}=0.48 a$, permittivity $\varepsilon_{2}=1.07-0.054 i$. The permeabilities of core and shell are both $\mu=1$. (c,d) show the real (black square line) and imaginary (blue triangle line) parts of effective permittivities and permeabilities corresponding to the eigenvalues. The dotted line indicates the real eigenvalue point with purely imaginary permittivity and permeability $\varepsilon_{e}=0.01838 i, \mu_{e}=-0.01838 i$. 
Therefore the electric field is

$$
E=\hat{z} E_{0} e^{i\left(k_{x} x+k_{y} y\right)} e^{-i \omega t} .
$$

Thus the magnetic field will be

$$
H=\hat{x} \frac{k_{x}}{\omega \mu_{x}} E_{0} e^{i\left(k_{x} x+k_{\|} y\right)} e^{-i \omega t}-\hat{y} \frac{k_{x}}{\omega \mu_{y}} E_{0} e^{i\left(k_{x} x+k_{\|} y\right)} e^{-i \omega t} .
$$

We can define the impedance as $Z_{\|}=\frac{E \cdot \hat{z}}{H \cdot \hat{y}}=-\frac{\omega \mu_{y}}{k_{x}}$, so the effective impedance of the PC is defined as $Z_{\|}^{\mathrm{PC}}=\frac{\int_{L} E^{\mathrm{PC}} \cdot \hat{z} d y}{\int_{L} H^{\mathrm{PC}} \cdot \hat{y} d y}$. $E^{\mathrm{PC}}$ and $H^{\mathrm{PC}}$ are obtained from the eigenfield at the incident boundary. According to the theory of PC energy band structure and the definition of the impedance, we can get the effective permittivity and permeability as

$$
\begin{aligned}
& \mu_{e}=\frac{k_{x}}{\mu_{0}} Z_{\|}, \\
& \varepsilon_{e}=\frac{k_{x}}{\omega \varepsilon_{0} Z_{\|}} .
\end{aligned}
$$

The real and imaginary parts of the eigenfrequency of our designed PC with a small Bloch wave vector along the $\Gamma X$ direction have been shown in Figs. 7(a) and 7(b), and then the effective permittivity and permeability of PC can be calculated by Eq. (B3) in Figs. 7(c) and 7(d). However, the effective parameters are not purely imaginary at the point of real eigenvalue. In order to accurately obtain the effective purely imaginary parameters, we fine-tune the permittivities of the PC from $\varepsilon_{1}=1.33+0.11 i, \varepsilon_{2}=1.07-0.054 i$ to $\varepsilon_{1}=1.154+0.11 i, \varepsilon_{2}=1.07-0.054 i$. After tuning, the band structure and the effective medium have been shown in Fig. 8, with almost purely imaginary effective parameters at the real eigenfrequency point, i.e., $\varepsilon_{e}=0.01838 i$, $\mu_{e}=-0.01838 i$. More interesting, the purely imaginary permittivity and permeability with the same absolute values but opposite signs, are the ZIMGL. Therefore, the ZIMGL has been achieved by the designed core-shell PC and the coherent perfect absorption and lasing can be demonstrated with in-phase $\left(a_{1}=a_{2}\right)$ and out-of-phase $\left(a_{1}=-a_{2}\right)$ incident waves.
[1] R. El-Ganainy, K. G. Makris, D. N. Christodoulides, and Z. H. Musslimani, Theory of coupled optical PT-symmetric structures, Opt. Lett. 32, 2632 (2007).

[2] C. E. Ruter, K. G. Makris, R. E. Ganainy, D. N. Christodoulides, M. Segev, and D. Kip, Observation of parity-time symmetry in optics, Nat. Phys. 6, 192 (2010).

[3] K. G. Makris, R. El-Ganainy, D. N. Christodoulides, and Z. H. Musslimani, Beam Dynamics in $P T$ Symmetric Optical Lattices, Phys. Rev. Lett. 100, 103904 (2008).

[4] S. Longhi, Bloch Oscillations in Complex Crystals with $P T$ Symmetry, Phys. Rev. Lett. 103, 123601 (2009).

[5] M. Kang, F. Liu, and J. Li, Effective spontaneous $P T$-symmetry breaking in hybridized metamaterials, Phys. Rev. A 87, 053824 (2013).

[6] P. Ambichl, K. G. Makris, L. Ge, Y. Chong, A. D. Stone, and S. Rotter, Breaking of PT Symmetry in Bounded and Unbounded Scattering Systems, Phys. Rev. X 3, 041030 (2013).

[7] A. Guo, G. J. Salamo, D. Duchesne, R. Morandotti, M. VolatierRavat, V. Aimez, G. A. Siviloglou, and D. N. Christodoulides, Observation of $\mathscr{P} \mathscr{T}$-Symmetry Breaking in Complex Optical Potentials, Phys. Rev. Lett. 103, 093902 (2009).

[8] T. Kottos, Broken symmetry makes light work, Nat. Phys. 6, 166 (2010).

[9] L. Feng, Y. L. Xu, W. S. Fegadolli, M. H. Lu, J. E. B. Oliveira, V. R. Almeida, Y. F. Chen, and A. Scherer, Experimental demonstration of a unidirectional reflectionless paritytime metamaterial at optical frequencies, Nat. Mater. 12, 108 (2013).

[10] S. Longhi, A unidirectionally invisible PT-symmetric complex crystal with arbitrary thickness, J. Phys. A: Math. Theor. 47, 485302 (2014).

[11] Y. Sun, W. Tan, H. Q. Li, J. Li, and H. Chen, Experimental Demonstration of a Coherent Perfect Absorber with PT Phase Transition, Phys. Rev. Lett. 112, 143903 (2014).
[12] Y. D. Chong, L. Ge, H. Cao, and A. D. Stone, Coherent Perfect Absorbers: Time-Reversed Lasers, Phys. Rev. Lett. 105, 053901 (2010).

[13] W. Wan, Y. D. Chong, L. Ge, H. Noh, A. D. Stone, and H. Cao, Time-reversed lasing and interferometric control of absorption, Science 331, 889 (2011).

[14] J. J. Luo, S. C. Li, B. Hou, and Y. Lai, Unified theory for perfect absorption in ultrathin absorptive films with constant tangential electric or magnetic fields, Phys. Rev. B 90, 165128 (2014).

[15] R. F. Oulton, V. J. Sorger, T. Zentgraf, R.-M. Ma, C. Gladden, L. Dai, G. Bartal, and X. Zhang, Plasmon lasers at deep subwavelength scale, Nature (London) 461, 629 (2009).

[16] H. Hodaei, M. A. Miri, M. Heinrich, D. N. Christodoulides, and M. Khajavikhan, Parity-time-symmetric microring lasers, Science 346, 975 (2014).

[17] S. Longhi, $P T$-symmetric laser absorber, Phys. Rev. A 82, 031801 (2010).

[18] Y. D. Chong, L. Ge, and A. D. Stone, PT-Symmetry Breaking and Laser-Absorber Modes in Optical Scattering Systems, Phys. Rev. Lett. 106, 093902 (2011).

[19] D. Dragoman, Complex conjugate media: Alternative configurations for miniaturized lasers, Opt. Commun. 284, 2095 (2011).

[20] A. Basiri, I. Vitebskiy, and T. Kottos, Light scattering in pseudopassive media with uniformly balanced gain and loss, Phys. Rev. A 91, 063843 (2015).

[21] Y. D. Xu, Y. Y. Fu, L. Xu, and H. Y. Chen, Conjugate metamaterials and the perfect lens, arXiv:1510.08638v1 [physics.optics].

[22] R. W. Ziolkowski, Propagation in and scattering from a matched metamaterial having a zero index of refraction, Phys. Rev. E 70, 046608 (2004).

[23] M. Silveirinha and N. Engheta, Tunneling of Electromagnetic Energy through Subwavelength Channels and Bends Using $\varepsilon$-Near-Zero Materials, Phys. Rev. Lett. 97, 157403 (2006) 
[24] A. M. Mahmoud and N. Engheta, Wave-matter interactions in epsilon-and-mu-near-zero structures, Nat. Commun. 5, 5638 (2014).

[25] S. Savoia, G. Castaldi, V. Galdi, A. Alù, and N. Engheta, Tunneling of obliquely incident waves through $P T$-symmetric epsilon-near-zero bilayers, Phys. Rev. B 89, 085105 (2014).

[26] X. Huang, Y. Lai, Z. H. Hang, H. Zheng, and C. T. Chan, Dirac cones induced by accidental degeneracy in photonic crystals and zero-refractive-index, Nat. Mater. 10, 582 (2012).

[27] Y. Wu, A semi-Dirac point and an electromagnetic topological transition in a dielectric photonic crystal, Opt. Express 22, 1906 (2014).

[28] J. Mei, Y. Wu, C. T. Chan, and Z.-Q. Zhang, First-principles study of Dirac and Dirac-like cones in phononic and photonic crystals, Phys. Rev. B 86, 035141 (2012).

[29] C. He, M.-H. Lu, X. Heng, L. Feng, and Y.-F. Chen, Paritytime electromagnetic diodes in a two-dimensional nonreciprocal photonic crystal, Phys. Rev. B 83, 075117 (2011).

[30] L. Feng, M. Ayache, J. Huang, Y. L. Xu, M. H. Lu, Y. Fainman, and A. Scherer, Nonreciprocal light propagation in a silicon photonic circuit, Science 333, 729 (2011).
[31] A. Regensburger, C. Bersch, M. A. Miri, G. Onishchukov, D. N. Christodoulides, and U. Peschel, Parity-time synthetic photonic lattices, Nature 488, 167 (2012).

[32] L. Ge, Y. D. Chong, and A. D. Stone, Conservation relations and anisotropic transmission resonances in one-dimensional $P T$ symmetric photonic heterostructures, Phys. Rev. A 85, 023802 (2012).

[33] Y. Wu, J. Li, Z.-Q. Zhang, and C. T. Chan, Effective medium theory for magnetodielectric composites: Beyond the longwavelength limit, Phys. Rev. B 74, 085111 (2006).

[34] L. Feng, Z. J. Wong, R.-M. Ma, Y. Wang, and X. Zhang, Singlemode laser by parity-time symmetry breaking, Science 346, 972 (2014).

[35] A. Mostafazadeh, Self-dual spectral singularities and coherent perfect absorbing lasers without PT-symmetry, J. Phys. A: Math. Theor. 45, 444024 (2012).

[36] A. Mostafazadeh, Point interactions, metamaterials, and PTsymmetry, Ann. Phys. 368, 56 (2016).

[37] J. Gear, F. Liu, S. T. Chu, S. Rotter, and J. Li, Parity-time symmetry from stacking purely dielectric and magnetic slabs, Phys. Rev. A 91, 033825 (2015). 